\title{
Itinerários terapêuticos de usuários de medicamentos de uma unidade de Estratégia de Saúde da Família
}

\author{
Therapeutic itineraries of users of medication \\ in a unit of the Family Heatlh Strategy
}

Giliane Dorneles Guerin ${ }^{1}$

Eloá Rossoni ${ }^{2}$

Denise Bueno ${ }^{3}$
${ }^{1}$ Escola de Saúde Pública do Rio Grande do Sul (ESP/

RS). Avenida Ipiranga 6311, Azenha. 90610-001 Porto

Alegre Rio Grande do Sul. giliane.guerin@yahoo.com.br ${ }^{2}$ Departamento de Odontologia Preventiva e Social, Faculdade de Odontologia, Universidade Federal do Rio Grande do Sul.

${ }^{3}$ Departamento de Produção e Controle de Medicamentos, Faculdade de Farmácia, Universidade Federal do Rio Grande do Sul (UFRGS).

\begin{abstract}
Therapeutic itineraries represent the trajectories taken by individuals in an attempt to resolve their health problems. The objective of this study was to analyze the trajectory when user prescription medication needs were not met in a Family Health Strategy Unit of the city of Porto Alegre. A database of users whose prescription needs were not fully met and the application of a questionnaire during home visits was performed. Users interviewed were between 53 and 85 years of age. The main problems reported were lack of money, physical difficulty in locomotion, side effects, illegible prescriptions, unavailability of medication in the local pharmacies of the city, fear of effects attributed to the medication, and "bureaucracy." When the medication is not available at the health unit, most users (60\%) reported buying it. With respect to the communication of the family health team in the orientation of ways that the user can gain access to the medication, $25 \%$ of the respondents reported that the team did not provide necessary information about the alternate location for the acquisition of the medication that was lacking.
\end{abstract}

Key words Therapeutic itineraries, pharmaceutical care, medication
Resumo Itinerários terapêuticos representam os caminhos percorridos por indivíduos na tentativa de solucionar seus problemas de saúde. Este trabalho analisou o percurso de usuários, quando não foram atendidas as necessidades prescritivas de medicamentos em uma Unidade de Estratégia de Saúde da Família de Porto Alegre, RS. Utilizou-se o cadastro de usuários que não tiveram suas necessidades prescritivas atendidas, efetuouse um questionário em visita domiciliar. Os entrevistados usuários tinham idade entre 53 e 85 anos. Os principais problemas relatados foram a falta de recursos financeiros, dificuldade física de locomoção, efeitos colaterais, receita médica não legivel, falta do medicamento nas Farmácias Distritais, receios relacionados a efeitos atribuídos ao medicamento, "burocracia". Quando não disponível o medicamento na unidade de saúde, a maioria dos usuários (60\%) referiu comprar. Com relação à comunicação da equipe de saúde da família na orientação dos fluxos que o usuário deveria acessar para adquirir o medicamento, $25 \%$ dos entrevistados referiram que a equipe não forneceu informações necessárias para a aquisição do medicamento que estava faltando.

Palavras-chave Itinerário terapêutico, Assistência farmacêutica, Medicamentos 


\section{Introdução}

O termo itinerário terapêutico pode ser utilizado como sinônimo de busca por cuidados terapêuticos, e procura descrever e analisar os caminhos percorridos por indivíduos na tentativa de solucionar o problema de saúde, considerando as práticas individuais e socioculturais ${ }^{1}$.

A preocupação sobre como as pessoas procuram ajuda para resolver suas demandas ou problemas de saúde, tem estado cada vez mais presente em estudos sobre planejamento, organização e avaliação de serviços assistenciais de saúde, uma vez que, os caminhos percorridos pelos usuários na busca por cuidados terapêuticos não necessariamente coincidem com esquemas ou fluxos pré-determinados pelo sistema de saúde ${ }^{2}$.

A saúde, a doença e o tratamento - apesar dos avanços tecnológicos - continuam sendo elementos influenciados diretamente por crenças, percepções, religiosidade, hábitos tradicionais e relações de poder. Neste contexto, a população circula entre os âmbitos populares e profissionais de saúde, construindo itinerários terapêuticos a partir de sua própria experiência em cuidados de saúde, (re) interpretando práticas e informações veiculadas no âmbito dos serviços de saúde, na comunidade e no modelo social vigente para conformar a utilização de medicamentos no cotidiano. Longe de ser exclusividade da área médica, as práticas de saúde, incluindo o uso de medicamentos e outros recursos terapêuticos, dependem da complexidade da realidade cultural em que se dão, imprimindo ao pensar e ao fazer diversas 'racionalidades' na gerência destes recursos ${ }^{3}$.

A busca do usuário ou de um grupo de indivíduos pela preservação ou recuperação de saúde pode mobilizar diferentes recursos, que foram classificados por Kleinman em Sistemas de Cuidado à Saúde. Estes sistemas se subdividem em três subsistemas: o Profissional, o Popular e o Folk ${ }^{4}$.

O subsistema Profissional é constituído pela medicina científica, demais profissões da saúde ou sistemas médicos alternativos profissionalizados (medicina chinesa, homeopatia) e representa a organização formal da prática de saúde. O subsistema Popular é aquele em que a família e o grupo social mais próximo desempenham papel importante. É um espaço eminentemente "leigo", onde a doença começa a ser definida e onde são desencadeados os vários processos terapêuticos de cura. Este subsistema também envolveria o campo leigo do tipo automedicação, conselhos de amigos, familiares e vizinhos e outras formas de assistência mútua. O subsistema folk ou paraprofissional, abrange todas as demais práticas de saúde "não profissionalizadas", como erveiros, benzedores, práticas religiosas e outras formas alternativas de cura. Assim, essa proposição de Kleinman fornece subsídios para a compreensão do papel dos medicamentos em diferentes cenários ${ }^{4}$.

Os usuários são o centro de redes terapêuticas, que estão conectadas às três alternativas do sistema de assistência à saúde. Recebida a orientação médica, o resultado poder ser a não adesão ao tratamento ou a transferência para outro segmento da rede terapêutica ${ }^{5}$. Temos a considerar que 70 a $90 \%$ dos episódios de doença são manejados fora do sistema formal de cuidado à saúde, ou seja, por autocuidado ou busca de formas alternativas de cura ${ }^{4}$.

A diversidade e a pluralidade de fatores que estão presentes na saúde e na doença são ainda portadores de múltiplos sentidos: pluralidade de condutas, pluralidade de terapeutas, pluralidade de etiologias (causas), pluralidade de percepções e de visões de mundo. Não se pode pensar na doença, suas causas e seu tratamento de forma linear onde só há lugar para uma forma de resolução do problema ${ }^{1}$.

Após obter a receita médica, o paciente se torna responsável pelo uso do medicamento. A orientação recebida do profissional prescritor é confrontada com outras fornecidas por diferentes profissionais da área da saúde, parentes, vizinhos e meios de comunicação de massa. A decisão do paciente é fortemente influenciada por valores culturais e fatores psicossociais, os quais podem levar ao uso incorreto desses produtos. Além de prejuízo para a saúde, o uso inadequado supõe um desperdício de recursos ${ }^{6}$.

Existe uma visão de atendimento à saúde organizada na lógica de que este deve ser simplesmente "usufruído" pelos usuários que assumiriam uma posição um tanto quanto passiva ${ }^{4}$. Devese considerar que é este usuário que fará a escolha final, ou seja, é ele quem detém o poder de cumprir ou não as determinações médicas ou procurar outras formas alternativas de cura.

O conhecimento sobre itinerários de pessoas em busca de atenção à saúde pode contribuir para a compreensão sobre o comportamento em relação ao cuidado e à utilização de serviços de saúde. A análise do itinerário terapêutico não pode se limitar a identificar a disponibilidade de serviços, seus modelos explicativos e a utilização que as pessoas fazem desses recursos - tais elementos são insuficientes para compreender o processo de busca por cuidado ${ }^{7}$. 
A hipótese de não cumprimento do itinerário terapêutico pode afastar o usuário do uso adequado do medicamento e consequentemente, comprometer a qualidade de vida e adesão ao tratamento proposto.

A Equipe de Saúde da Família assume papel de fundamental relevância no diagnóstico comunitário, qualificando determinadas situações (individuais ou comunitárias) como problema ou não-problema de saúde e, portanto, desempenhando papel primordial para promover ou impossibilitar a realização de determinadas trajetórias na busca pelo cuidado em saúde ${ }^{7}$.

A escolha da temática Itinerários terapêuticos de usuários de medicamentos em uma Estratégia de Saúde da Família surgiu como processo questionador das formas de busca de cuidados pelo usuário diante do processo de adoecimento (relacionadas ao seu contexto pessoal, familiar, cultural, dentre outros), e incluiu uma análise das facilidades, dificuldades e limitações quanto ao acesso aos medicamentos que não foram disponibilizados pela Unidade de Saúde de referência.

Algumas questões foram levantadas como possíveis interferentes no cumprimento do itinerário terapêutico dos usuários: o deslocamento territorial significativo pode ser uma barreira no cumprimento da terapia proposta? O usuário faz uso de automedicação como forma de compensar a falta do medicamento na unidade de saúde acessada? O custo do medicamento, quando este não é disponibilizado por meio público, interfere no cumprimento do tratamento preconizado? A possível falta de comunicação entre a equipe de saúde e o usuário pode estar dificultando o cumprimento do itinerário terapêutico? (Quadro 1).

Este trabalho, realizado como requisito para a conclusão do Programa de Residência Integrada da Escola de Saúde Pública do Rio Grande do Sul focaliza a compreensão dos itinerários terapêuticos como ferramenta para qualificação da assistência, uma vez que, associa aspectos sobre o acesso, a utilização dos serviços e os fatores relacionados ao contexto do usuário.

Supõe-se que a racionalização no uso destes recursos pode resultar em otimização de resultados terapêuticos, aumento da adesão ao tratamento e consequentemente em redução dos custos de atendimentos.

O objetivo do estudo foi analisar o percurso do usuário, quando não foram atendidas as necessidades prescritivas de medicamentos em uma Unidade de Estratégia de Saúde da Família pertencente ao Distrito Partenon/Lomba do Pinheiro, no município de Porto Alegre, RS.

A motivação em realizar esta pesquisa surgiu a partir do interesse em analisar o medicamento prescrito, quando ausente, interferindo na relação de cuidado e utilização de serviços de Atenção à Saúde.

\section{Métodos}

O delineamento deste estudo é qualitativo e foi inspirado nos estudos culturais, os quais anali-

Quadro 1. Causas que dificultaram o cumprimento do itinerário terapêutico.

\begin{tabular}{|c|l|}
\hline \multicolumn{1}{|c|}{ Causas } & \multicolumn{1}{c|}{ Falas dos usuários } \\
\hline $\begin{array}{c}\text { Receita médica } \\
\text { ilegível }\end{array}$ & $\begin{array}{l}\text { Eu uso os medicamentos dessa receita velha de } 2007 \text { porque é a única que eu consigo } \\
\text { entender o que diz (U1) }\end{array}$ \\
\hline Falta de dinheiro & $\begin{array}{l}\text { O XX é muito caro, tem vezes que não posso usar ele porque não tenho condições } \\
\text { de comprar, porque tenho outras despesas com minha filha e minha netinha e aí não } \\
\text { sobra o dinheiro (U5) }\end{array}$ \\
\hline $\begin{array}{c}\text { Fificuldade física do } \\
\text { medicamento na tue né, tenho muita dificuldade para caminhar, moro sozinho, nem sempre os } \\
\text { familiares estão por perto e aí não tenho como conseguir o remédio, daí fico sem } \\
\text { Farmácia Distrital }\end{array}$ & $\begin{array}{l}\text { Se não tem no Bananeiras, vou no Modelo. Se não tem também, daí eu fico sem o } \\
\text { medicamento. Várias vezes eu fiquei sem o medicamento porque não tem, daí o quê } \\
\text { que eu vou fazer, né? E aí não deu para comprar e fiquei sem (U17) }\end{array}$ \\
\hline $\begin{array}{c}\text { Efeitos colaterais } \\
{[\ldots] \text { quando eu uso essa XX eu tenho sempre diarréia, e aí eu paro de tomar por uns }} \\
\text { dias e quando sinto que o açúcar sobe eu volto a tomar (U8) }\end{array}$ \\
\hline $\begin{array}{c}\text { Medo do efeito do } \\
\text { medicamento }\end{array}$ & $\begin{array}{l}\text { O médico me deu o XX para dormir, mas não uso porque tenho medo de entrar em } \\
\text { coma a noite e não ter ninguém para me socorrer (U10) }\end{array}$ \\
\hline
\end{tabular}


sam os significados que as pessoas atribuem às suas vivências cotidianas.

A entrada em campo e o início do contato com informantes merecem atenção especial por caracterizar o começo de uma relação que pretende ser de confiança ${ }^{8}$.

A amostra populacional deste estudo foram usuários de medicamentos que não tiveram suas prescrições de medicamentos integralmente atendidas pela unidade de estratégia de saúde da $\mathrm{Fa}$ mília Herdeiros, pertencente ao distrito sanitário Partenon/ Lomba do Pinheiro, localizada na cidade de Porto Alegre (RS) no período de março a agosto de 2011.

A produção de dados foi realizada em dois momentos. Inicialmente, foi realizado um levantamento dos usuários que não acessaram integralmente o(s) medicamento(s) na unidade de estratégia de saúde da família. Efetuou-se um banco de dados no programa Microsoft Excel ${ }^{\circledR}$ contendo o nome do paciente, o número do prontuário, o endereço, a data da prescrição, o local de origem da prescrição, os medicamentos não atendidos e a dosagem. Partindo-se deste banco de dados, realizou-se a segunda etapa da produção de dados, que consistiu na realização de visitas domiciliares para 20 usuários, selecionados aleatoriamente do banco de dados, onde foi aplicado um questionário semiestruturado, gravada a entrevista e registradas observações no diário de campo.

Como parte integrante do processo metodológico, houve transcrição das entrevistas, análise dos transcritos, dos registros do diário de campo e do questionário semiestruturado.

Neste estudo utilizou-se a análise do conteúdo por categorização aplicando a análise temáti$\mathrm{ca}$, que consiste em isolar temas do texto e extrair as partes utilizáveis, de conformidade com o problema pesquisado9 .

A partir dos dados produzidos neste estudo foram propostas as seguintes categorias de análise: causas do não cumprimento do itinerário terapêutico, comunicação da equipe de Saúde da Família com o usuário, automedicação como forma de compensar o medicamento não acessado e simbolismo do medicamento.

O projeto de pesquisa foi aprovado pelo Comitê de Ética da Secretaria Municipal de Saúde de Porto Alegre/RS.

\section{Resultados e discussão}

Foram realizadas 20 visitas domiciliares entre os meses de março a agosto de 2011. Dos entrevistados, $80 \%$ eram mulheres com faixa etária que variou 53 a 85 anos e $20 \%$ eram homens com faixa etária que variou de 58 a 73 anos. Quanto ao grau de escolaridade dos entrevistados prevaleceu $(60 \%)$ como formação o ensino fundamental incompleto. A renda familiar declarada em $80 \%$ das entrevistas foi um salário mínimo.

Dos usuários, 20\% tiveram um medicamento não fornecido pela Unidade de Saúde da Família (USF), 30\% dos usuários tiveram dois medicamentos não fornecidos pela USF, 30\% dos usuários tiveram três medicamentos não fornecidos pela USF, $5 \%$ dos usuários tiveram quatro medicamentos não atendidos pela USF, 10\% dos usuários tiveram cinco medicamentos não fornecidos pela USF, e 5\% dos usuários tiveram seis medicamentos não fornecidos pela USF, conforme Tabela 1. O tempo médio de duração das entrevistas foi de 40 minutos.

\section{Simbolismo do medicamento}

Quem ébonita não precisa de remédio [...] (U15) O medicamento abarca um conjunto de simbolismos que ultrapassa o seu valor farmacológico e influencia diretamente suas formas de utilização. Os valores e os significados que lhe são atribuídos, o símbolo de saúde, materializado e ao alcance por meio do acesso aos medicamentos, é o mais notável e reconhecido pela sociedade. No entanto, também pode simbolizar a própria doença e a condição de doente, dificultando o seguimento dos tratamentos. Iniciar um tratamento medicamentoso significa aceitar a própria doença $^{10}$.

$\mathrm{O}$ efeito de qualquer medicamento sobre um indivíduo depende de uma série de elementos adicionais às suas propriedades farmacológicas, que podem ser exemplificados como: as características da droga (gosto, formato, cor, nome), características do usuário que recebe a droga (experiência anterior, escolaridade, personalidade, contexto sociocultural), características da pessoa que prescreve a droga (status profissional, senso de autoridade, personalidade), ambiente no qual a droga é administrada (consultório médico, hospital, laboratório, ocasião social $)^{5}$

[...] Minha saúde não é melhor porque acho que tô tomando remédio demais ... o meu filho disse: antes a senhora não tomava nada e tava bem, tava sempre trabalhando... (U18) 
Tabela 1. Características dos entrevistados (usuários de medicamentos) segundo sexo, idade, escolaridade, renda familiar e número de medicamentos que não foram acessados na Unidade de Saúde da Família, Porto Alegre, 2011.

\begin{tabular}{|c|c|c|c|c|c|}
\hline Usuário & Sexo & $\begin{array}{l}\text { Idade } \\
\text { (anos) }\end{array}$ & Escolaridade & Renda Familiar & $\begin{array}{l}\text { Quantidade de medicamentos } \\
\text { não fornecidos }\end{array}$ \\
\hline $\mathrm{U} 1$ & M & 73 & $1^{\circ}$ grau incompleto & 900 reais & 4 \\
\hline $\mathrm{U} 2$ & $\mathrm{~F}$ & 85 & Não estudou & 1 salário & 3 \\
\hline U3 & M & 61 & $1^{\circ}$ grau incompleto & 1 salário & 2 \\
\hline $\mathrm{U} 4$ & $\mathrm{~F}$ & 53 & $1^{\circ}$ grau incompleto & 1 salário & 1 \\
\hline U5 & $\mathrm{F}$ & 75 & $1^{\circ}$ grau incompleto & 1 salário & 3 \\
\hline U6 & $\mathrm{F}$ & 55 & Não estudou & 1 salário & 3 \\
\hline U7 & $\mathrm{F}$ & 71 & $1^{\circ}$ grau incompleto & 1 salário & 3 \\
\hline U 8 & M & 58 & $1^{\circ}$ grau incompleto & Desempregado & 1 \\
\hline U9 & $\mathrm{F}$ & 57 & Não estudou & 1 salário & 5 \\
\hline $\mathrm{U} 10$ & M & 60 & $1^{\circ}$ grau completo & 1 salário & 2 \\
\hline $\mathrm{U} 11$ & $\mathrm{~F}$ & 75 & $2^{\circ}$ grau completo & 1 salário & 5 \\
\hline $\mathrm{U} 12$ & $\mathrm{~F}$ & 53 & $1^{\circ}$ grau completo & 1 salário & 2 \\
\hline U 13 & $\mathrm{~F}$ & 83 & Não estudou & 1 salário & 1 \\
\hline U 14 & $\mathrm{~F}$ & 73 & $1^{\circ}$ grau incompleto & 1 salário & 3 \\
\hline U 15 & $\mathrm{~F}$ & 68 & $1^{\circ}$ grau incompleto & 1 salário & 1 \\
\hline U16 & $\mathrm{F}$ & 66 & $1^{\circ}$ grau incompleto & 4 salários & 2 \\
\hline $\mathrm{U} 17$ & $\mathrm{~F}$ & 65 & $1^{\mathrm{o}}$ grau incompleto & 1 salário & 6 \\
\hline $\mathrm{U} 18$ & $\mathrm{~F}$ & 62 & $1^{\circ}$ grau incompleto & 1 salário & 3 \\
\hline U19 & $\mathrm{F}$ & 78 & $2^{\circ}$ grau incompleto & 1 salário & 2 \\
\hline $\mathrm{U} 20$ & $\mathrm{~F}$ & 70 & $1^{\circ}$ grau completo & 2 salários & 2 \\
\hline
\end{tabular}

[...] meu companheiro faleceu aos 56 anos de HIV porque não tomava os medicamentos, teve complicações. Ele não falava pra ninguém que era doente... Tinha medo de ser rejeitado [..] (U15)

Pode-se evidenciar na fala da usuária 18 (U18), o status da utilização do medicamento como símbolo de uma limitação para o trabalho. Com relação à fala da usuária 15 (U15), evidencia-se o fato de que, muitas vezes, o desejo de não tomar o medicamento pode estar representando uma forma de negar a própria doença, uma vez que, utilizar o medicamento cronicamente pode representar o encontro diário com a doença, com a condição inegável de doente.

Compreender a forma como o medicamento é entendido e como o usuário se relaciona com ele é etapa essencial para que os profissionais de saúde possam abordá-lo de forma efetiva, apesar de ser este um tema raramente abordado na terapêutica ${ }^{10}$.

Apesar de 100\% dos usuários entrevistados afirmarem que sempre conseguiram adquirir $o(s)$ medicamento(s) não encontrado na Unidade de Saúde da Família acessada, observou-se que tal afirmação não se confirmava no decorrer da maioria dos discursos. E com isso, foram re- latadas as causas que muitas vezes dificultaram o cumprimento do itinerário terapêutico.

A experiência de cada sujeito é permanentemente definida e modificada pelo agir cotidiano e pela busca de soluções práticas para os processos de adoecimento e não pode ser sintetizado em um sistema coerente e ordenado de ideias. Além disso, as formas como os sujeitos explicam tais processos não mostram coerência com as ações que desenvolvem ${ }^{11}$.

\section{A peregrinação no acesso ao medicamento}

É muita burocracia para adquirir os medicamentos [...] (U4)

Trazer a "peregrinação" de usuários em busca por resolver necessidades em saúde pode pôr em evidência as implicações dos sistemas de saúde em seus itinerários, aquilo que esses sistemas possibilitam de resolutividade, ou seja, o que dão conta e tudo aquilo que "escapa" aos mesmos, bem como os efeitos que produzem sobre a experiência de usuários e famílias ${ }^{12}$

Quando não disponível o medicamento na unidade de saúde, a maioria dos usuários $(60 \%)$ referiu comprar. 
Me senti humilhada uma vez que fui pegar meus remédios comecei a passar mal, ter crises de falta de ar e não tive preferência no atendimento (U9)

Eu tenho que sair daqui de casa, eu tenho que subir lá no posto para pegar receitas.. Daí tu não encontra num, vai no outro [...] Estou sempre correndo atrás desses remédios para não faltar (U17)

[...] a burocracia para adquirir as coisas na Farmácia do Estado desanima (U10)

[...] a coisa mais difícil que eu acho quando tenho que buscar meus remédios é a demora desse ônibus... (U11)

Aqui temos reproduzido algumas falas de sofrimento, desabafo, insatisfação de alguns usuários no acesso ao medicamento. Temos a considerar, que muitas vezes, esta indignação, descontentamento do usuário frente a suas experiências, muitas vezes negativa, poderá conduzir o usuário para a judicialização como forma de "adquirir" os seus direitos.

Quando um usuário busca acesso a um serviço, ele tem um único objetivo, que é solucionar o seu problema de saúde. Para alcançar este objetivo, ele percorrerá inúmeros caminhos, ainda que imprevistos para o sistema de saúde, criando para si o itinerário terapêutico mais apropriado. Por exemplo, um usuário que resolve acessar seus medicamentos via judicial porque acredita que a unidade de saúde do seu bairro não tem todos os medicamentos que ele toma, ou porque frequentemente estes medicamentos estão em falta ou porque ele não se sente acolhido pela sua unidade de saúde de referência.

No estudo de Lima ${ }^{13}$ todos os usuários entrevistados, iniciaram sua peregrinação nas unidades de saúde, e como não encontraram respostas para as suas necessidades de tratamento, ou seja, na ausência do medicamento na unidade de saúde, recorreram à via judicial.

As alternativas legais para a propositura de ações judiciais relacionadas ao direito à saúde, contra os poderes públicos, permitem vários ângulos de observação deste fenômeno. Todavia, os estudos sobre o tema apontam que grande parte desta demanda se concentra nos processos judiciais individuais de cidadãos reivindicando o fornecimento de medicamentos. É consenso que o uso da via judicial para o fornecimento de medicamentos presentes nas listas oficiais públicas (as listas de medicamentos essenciais como aquelas que contêm um elenco selecionado para o fornecimento pelo SUS, sejam elas as do componente de financiamento, pactuado entre as instâncias, ou ampliadas/reduzidas pelos gestores locais) é uma forma legítima de garantir o pleno exercício do direito à assistência individual terapêutica, que integra o direito à saúde na lei brasileira ${ }^{14}$.

Algumas características comuns são identificadas nos estudos realizados em diferentes regiões do país. A maioria dos pedidos é individual e tem sido deferida tendo como praticamente única base a prescrição medicamentosa apresentada pelo reivindicante. A segunda característica é o fato de a prescrição conter tanto medicamentos incorporados como não incorporados pela assistência farmacêutica (AF) do SUS, alguns sem registro no país ou em indicação terapêutica não constante do registro sanitário. A terceira característica é o crescimento exponencial das demandas judiciais e dos gastos com medicamento ${ }^{14}$.

\section{Não cumprimento do itinerário terapêutico...}

Paniz e colaboradores referem que a falta de acesso a medicamentos para o tratamento de enfermidades pode levar ao agravamento do quadro e aumento dos gastos com atenção secundária e primária ${ }^{15}$.

Eu fiquei uns dias sem esse remédio do coração e eu não tinha dinheiro para comprar ... daí passei mal, sabe...fui parar no hospital com dor muito forte, daí me fizeram cateterismo (U17)

Teve um mês que minha filha não pôde comprar o meu remédio e acabei tendo complicações graves de saúde e fui internada (U2)

Eu fiquei sem conseguir comprar o remédio XX, e na Farmácia Bananeiras só dão esse remédio para as grávidas. Daí fiquei um tempo sem e passei mal, fui internada (U15)

\section{Autocuidado...}

Fiquei um mês sem usar os remédios para pressão, mas aí tomei o chá de XX que também é bom para pressão (U5)

Às vezes substituo o YY pelo chá de folhas de XX que é bom para pressão (U6)

A população constrói seus itinerários terapêuticos a partir de sua própria experiência em cuidados de saúde. Tais dimensões não devem ser negligenciadas pelas equipes de saúde, devem fazer parte das estratégias de cuidado, de forma a tornar reconhecimento das experiências singulares o motor da construção de projetos de cuidado $^{10}$.

Podemos perceber que a população possui saberes que nem sempre estão validados academicamente e diante disto, nem sempre o profis- 
sional de saúde está preparado para lidar com as diferentes práticas de saúde. Cabe ao profissional, encaminhar estes casos para discussão em equipe.

Muitos profissionais entendem que a solução para todos os males está na medicalização e no saber comprovado cientificamente. Ao legitimarem terapêuticas científicas, outorgam às práticas resultantes da medicina convencional a única possibilidade de êxito na saúde do usuário. Julgam seus saberes como soberanos e nem cogitam outra possibilidade de tratamento, despersonalizando o indivíduo. O processo de medicalização tem na medicina científica o seu fio condutor e indutor, ignorando a individualidade do paciente face ao seu caráter curativo, ou mesmo preventivo, através da ênfase na doença ${ }^{16}$.

Ceccim destaca a importância da "entre-disciplinaridade" nas práticas de saúde, diferenciando-a da interdisciplinaridade, por ser "mais que uma intersecção entre os profissionais", mas por emergir do entre. O autor propõe a "entredisciplinaridade" como forma de compreender o trabalho multiprofissional e interdisciplinar, "um lugar de sensibilidade e equilíbrio metaestável, em que a prática terapêutica emergiria em clínica mestiça ou clínica nômade; em que todos os potenciais seguiriam se atualizando e o equilíbrio não seria outro que não a transformação permanente de si, dos entornos, do trabalho"17. Nesta nova visão, o cuidado será realizado entre os profissionais da equipe sem delimitação de fronteiras entre as profissões, resultando num projeto terapêutico usuário-centrado.

\section{Comunicação da equipe de saúde da família} (U1)

Só me falaram que não tinha meu remédio...

A equipe de saúde da família desempenha papel primordial para promover ou impossibilitar a realização de determinadas trajetórias na busca pelo cuidado em saúde. Uma das condições para uma assistência de qualidade e segura é que o sistema possua um canal de comunicação eficaz, permitindo às equipes transmitir e receber informações de forma clara e correta ${ }^{7}$.
Com relação aos usuários entrevistados, $25 \%$ referiram que a equipe não referenciou o local para aquisição do medicamento que não estava disponível. Verificou-se que muitos dos medicamentos que os usuários estavam comprando estão disponíveis na rede pública.

\section{Considerações finais}

Compreender os fatores que estão dificultando o cumprimento do itinerário terapêutico é uma forma de acolher o usuário e contribuir para promoção da saúde, reduzindo o número de internações hospitalares, de judicialização de medicamentos por quebra de itinerário terapêutico, entre outros problemas enfrentados. Quando o indivíduo não consegue seguir seu plano terapêutico pré-estabelecido para a aquisição dos medicamentos, uma demanda de saúde pode ser gerada, causando a curto e longo prazo consequências que irão refletir no sistema de atenção individual e coletivo.

A utilização de medicamentos é um processo complexo, pois envolve múltiplos determinantes, diferentes atores sendo influenciada por fatores de natureza cultural, social, econômica e política ${ }^{18}$. Diante disto, as ações e políticas relativas ao fornecimento e utilização de medicamentos deverão contemplar estes aspectos, confrontando as expectativas dos usuários, os aspectos econômicos e os critérios técnico-científicos para a tomada de decisões.

É necessário que a equipe de saúde esteja atenta aos usos e costumes culturais da comunidade atendida. Como não devem existir atores privilegiados na produção de saúde, nem preconceitos ou hierarquizações, importante se faz o entendimento e a valorização das formas de tratamento e cura, no contexto em que se desenvolvem.

Com base no exposto, verificou-se que os estudos com itinerários terapêuticos são úteis para a qualificação da assistência, planejamento, gestão, pois promovem reflexões acerca da relação do usuário com o profissional de saúde e com o sistema de saúde. Sendo assim, centrar as ações no usuário e na sua cultura, promove humanização do cuidado e integralidade na atenção à saúde. 


\section{Referências}

1. Gerhardt TE. Itinerários terapêuticos em situações de pobreza: diversidade e pluralidade. Cad Saude Publica 2006; 22(11):2449-2463.

2. Cabral ALLV, Martinez-Hemáez A, Andrade EIG, Cherchiglia ML. Itinerários terapêuticos: o estado da arte da produção científica no Brasil. Cien Saude Colet 2011; 16(11):4433-4442.

3. Leite SN, Vasconcellos MP. Medicamentos e outros Recursos Utilizados no Enfrentamento das Doenças Infantis no Cotidiano Familiar: Estudo Qualitativo. Latin American Journal of Pharmacy 2009; 28(6): 835-842

4. Oliveira FA. Antropologia nos serviços de saúde: integralidade, cultura e comunicação. Interface Comum Saúde Educ 2002; 6(10):63-74.

5. Helman CG. Cultura, Saúde e Doença. Porto Alegre: Artes Médicas; 1994.

6. Lage EA, Freitas MI, Acurcio FA. Informação sobre medicamentos na imprensa: uma contribuição para o uso racional? Cien Saude Colet 2005; 10(Supl.):133139.

7. Pontes ALM, Furtado S, Martins C, Matta GC, Morosini MVCG. Itinerários terapêuticos e Estratégia de Saúde da Família: discursos sobre o processo saúde-doença e acesso aos serviços de saúde no trabalho do Agente Comunitário de Saúde. In: Pinheiro R, Silva Junior AG, Mattos RA. Atenção básica e integralidade: contribuições para estudos de práticas avaliativas em saúde. Rio de Janeiro: Abrasco; 2008. p. 237.

8. Leite SN, Vasconcellos MP. Construindo o campo da pesquisa: reflexões sobre a sociabilidade estabelecida entre pesquisador e seus informantes. Saúde e Sociedade 2007; 16(3):169-177

9. Richardson RJ. Pesquisa social: métodos e técnicas. $3^{a}$ Edição. Revisada e ampliada. São Paulo: Atlas; 1999.

10. Leite SN, Vasconcellos MP. Os diversos sentidos presentes no medicamento: elementos para uma reflexão em torno de sua utilização. Arquivos Catarinenses de Medicina 2010; 39(3):18-23.

11. Mangia EF, Murato MT. Itinerários terapêuticos e construção de projetos terapêuticos cuidadores. Rev. Ter. Ocup. Univ. 2008; 19(3):176-182.
12. Bellato R, Araújo LFS, Castro P. O itinerário terapêutico como uma tecnologia avaliativa da integralidade em saúde. In: Pinheiro R, Silva Junior AG, Mattos RA, organizadores. Atenção básica e integralidade: contribuições para estudos de práticas avaliativas em saúde. Rio de Janeiro: Abrasco; 2008. p. 167.

13. Lima VMCL. Peregrinação, empoderamento, retrocessos no acesso a medicamentos por via judicial no Estado do Amazonas - Brasil [dissertação]. Itajaí: Universidade do Vale do Itajaí; 2009.

14. Pepe VLE, Figueiredo TA, Simas L, Osorio-de-Castro CGS, Ventura M. A judicialização da saúde e os novos desafios da gestão da assistência farmacêutica. Cien Saude Colet 2010; 15(5):2405-2414.

15. Paniz VM, Fassa AG, Facchini LA, Bertoldi AD, Piccini RX, Tomasi E, Thumé E, Silveira DS, Siqueira FV, Rodrigues MA. Acesso a medicamentos de uso contínuo em adultos e idosos nas regiões Sul e Nordeste do Brasil. Cad Saude Publica 2008; 24(2):267-280.

16. Junges JR, Barbiani R, Ávila Soares N, RBP Fernandes, Lima MS. Saberes populares e cientificismo na estratégia saúde da família: complementares ou excludentes? Cien Saude Colet 2011; 16(11):4327-4335.

17. Ceccim RB. Equipe de saúde: perspectiva entredisciplinar na produção dos atos terapêuticos. In: Pinheiro R, Mattos RA, organizadores. Cuidado: as fronteiras da integralidade. 3a Edição. Rio de Janeiro: IMS, Uerj, Abrasco; 2006. p. 259-278.

18. Heckler APM, Oliveira FA. In: Pinheiro R, Mattos RA, organizadores. Cuidar do cuidado: responsabilidade com a integralidade das ações de saúde. Rio de Janeiro: Abrasco; 2008.

Artigo apresentado em 30/04/2012

Aprovado em 17/07/2012

Versão final apresentada em 30/08/2012 